\title{
Analogies as Creative Inspiration Sources in the Design Studio: The Teamwork
}

\author{
By Hernan Casakin* \\ Arjan van Timmeren ${ }^{\dagger}$
}

\begin{abstract}
Analogical reasoning can be seen as an important aid in problem solving, and architectural design problem solving in particular. Analogical reasoning was found to be an important aid supporting architectural education. In this paper, we explored the use of visual and verbal analogies in the early phases of the design process when student designers work alone and as a team. In addition, we investigated the contribution of different types of analogy on the creativity of the final design outcome. In an empirical study the use of visual and verbal analogies were compared in regard to the question of how designers deal with different problem solving activities during the design process. Findings showed that the help provided by visual analogy was mainly successful in a definition of the problem, clarifications of ideas, and evaluations of solutions. Verbal analogy, on the other hand, was particular successful in generating design ideas. Both types of analogies contributed to the originality and aesthetic value of the final design outcome. Independently of the type of analogy used, working in team was found to be fundamental to enrich different aspects of the design activity. Implications for design education are discussed.
\end{abstract}

\section{Introduction}

Analogy is a cognitive process that can support the acquisition of new knowledge (Hofstadter, 2001; Holyoak and Thagard, 1997), and aid in learning (Brown, 1989). Analogical reasoning helps to understand unfamiliar situations according to familiar ones. It can be seen as reconceptualization (Kuhn, 1971) to support change of perception and evaluation of known data. Analogy makes possible to identify, map and transfer structural information from a known situation (referred to as the source), to a situation requiring elucidation and explanation (referred to as the target) (Holyoak and Thagard, 1995). An analogy is established when a correspondence is identified among known relations between entities in the source, and possible relations between entities in the target (e.g., Bearman et al., 2007). An analogy can be compared to a similarity, as they both entail an alignment of relational structure. However,

${ }^{*}$ Senior Lecturer, Ariel University, Israel.

${ }^{\dagger}$ Professor, Delft University of Technology, The Netherlands. 
whereas in an analogy only relational predicates are shared, in literal similarity both relational predicates and object attributes are shared (Gentner, D. \& Markman, 1997). Due to its abstracting power, this tool can contribute to create new concepts, which in turn can help to identify more remote analogies, from which it is possible to discover even more abstract concepts (Holyoak \& Taghard, 1996).

Analogical reasoning is seen as an effective tool in problem-solving (Dunbar and Blachette, 2001), and an essential part of the creative process (Koestler, 1964). An analogy facilitates access to familiar solution principles that can aid to solve unknown problems. Establishing correspondences between two problems, and transferring a solution from the source problem to the novel one is vital for the employment of a suitable analogy.

The use of analogy is of large importance in design problem solving, characterized by the existence of vague initial requirements, not fully specified goals, and the lack of operators or algorithms to generate suitable design solutions (Simon, 1984). Analogical reasoning can help generate unexpected solutions, as demanded by the design problems. The aid provided by these tools in design has been explored in a number of studies (Ball et al. 2004; Ball and Christensen, 2009; Casakin, 2010; Casakin and Goldschmidt, 2000; Casakin, 2004). A goal of most of these works was to explore how it could be possible to help designers enhance their skills in design problem solving, and how they could be aided to use critical prior knowledge to deal with novel design problems.

The use of analogy is particularly prolific in architecture. There are a large number of examples in the design literature of famous architects employing successful analogies. Illustrative examples include the use that Le Corbusier made of a picture of the Cunard Ocean Liner as an analogical source for the design of the Unite d'Habitation building in Marseille, or the analogy that this architect established with a snail shell when he designed the Museum of Tokyo (See Figure1).

Analogical reasoning is common in the early stages of the design process. Characteristic in this stage of the process is the development of concepts and ideas which can affect many of the design decisions taken during the later stages of the process (Goldschmidt and Smolkov, 2006). At this conceptual stage, a large part of the design activity consists in the exploration of external stimuli that can serve as analogical sources. Stimuli are considered to be convenient sources of inspiration since they can contribute to enhance design thinking, and aid in idea generation (Cardoso and Badke-Schaub, 2011). Such analogical sources can endow the designer with an initial point of departure into the problem solving process (Casakin, 2004; Goldschmidt, 1995) to explore novel ideas (Ball. et al., 2009), restructure and reflect on design situations, and enhance innovation (Visser, 1996). External analogical sources may contain important search cues to access information stored in memory, and can influence the generation of new creative ideas (Cai et al., 2010; Perttula and Sipila, 2007). Idea generation is possible due to the identification of emergent visual clues, which is supported by the interactive dialogue that 
designers establish between the available external sources and their internal representations (Goldschmidt, 1994).

Figure 1. Example of the Use of Analogy in Design by Le Corbusier. a) Snail Shell (Source); b) Tokyo Museum (Target)

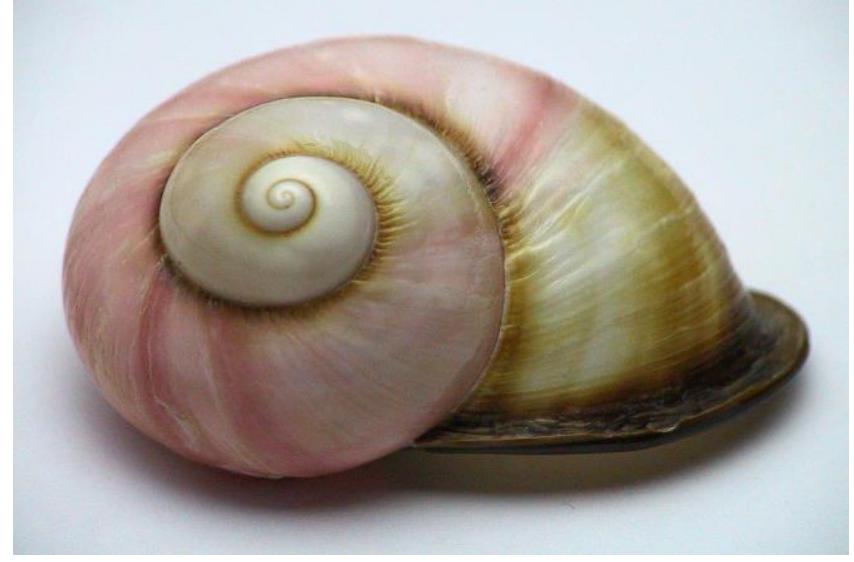

(a)
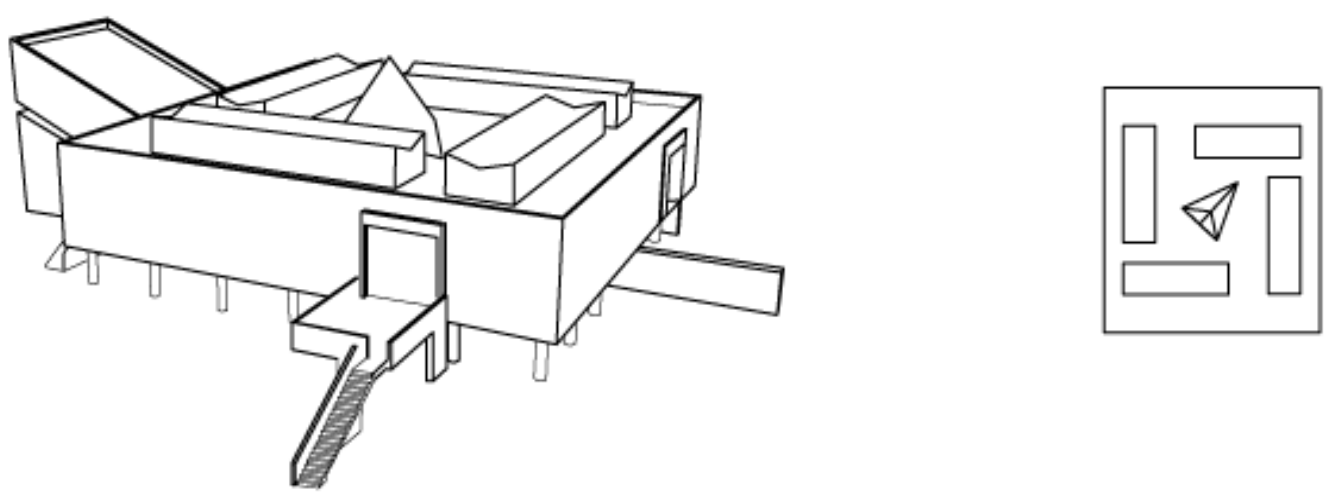

(b)

Idea generation can be triggered by different types of sources, but in architectural design there is a marked predilection for the use of visual sources (Hannington, 2003). Different types of images such as drawings, photographs, objects, or free-hand sketches are recurrently used as problem solving aids.

In both design practice and education, the use of external sources, visual stimuli in particular, can be helpful to enhance the quality of design solutions (Goncalves et al., 2014), improve design knowledge and skills (Cai et al., 2010), and enhance creativity (Casakin, 2012). Creative cognition processes related to analogizing are decisive in the production of innovative outcomes. In this process, designers are able to restructure the design problem from innovative perspectives (Schon, 1966) and establish analogical relations with the design problem. Making abstractions and cross-domain generalizations 
while transferring analogical principles enables transcending conventional knowledge to produce creative designs.

Visual analogies are largely used in design. This is partly due to the large number of visual sources that designers employ when solving design problems. During the idea generation process, designers can identify visual stimuli as potential analogs, and generate a mapping and transfer of analogical principles through structural or surface relations that are graphically established. Goldschmidt (1995) suggested that establishing correspondences between the visual sources and the design problem can contribute in the successful organization of the design into a coherent structural system.

In addition to visual aids, verbal/text stimuli were also found to be an efficient mechanism in problem solving. Goldschmidt and Litan Sever (2011) studied the use of text as a tool to inspire design ideas. They showed that designs based on text-stimuli were assessed as more original compared to those designs produced without any kind of stimuli. These researchers concluded that reading text containing ideas can be inspiring, and this can contribute to enhance the originality and creativity of the designs. They proposed that text can be useful as a pedagogical approach in the design studio.

Goncalves et al. (2014) explored what kind of stimuli designers with different levels of expertise use as inspirational sources during the idea generation process. These researchers showed that both novices and experts use images as stimuli for idea generation more frequently than text. On the other hand, novices tended to rely more on textual representations than experts. Malaga (2000) carried out an experiment where subjects were exposed to words, pictures, and combined words and images, and were requested to produce ideas to solve a task. He showed that the use of visual images lead to more creative ideas than text or combined stimuli. We have claimed that the use of analogy and visual analogy in particular, plays a major function in the generation of new ideas in design problem solving. But despite its importance, not much is known regarding how designers use stimuli, either visual or verbal, as analogical sources to deal with different design activities, and how these tools affect idea generation. On the other hand, no empirical study has compared simultaneously the use of external stimuli as visual and verbal analogical sources in design.

Another aspect of the present study is concerned with how analogical reasoning affects the design activity of both the individual designers and the design teams. The variety of thinking among members of design teams can be maximized and integrated upon through the establishment of shared knowledge across individuals, so that they could deal effectively with the intrinsic ambiguity of ill-defined design problems (Goel and Pirolli, 1992; Simon, 1984). Thus, a main goal for design teams consists in interacting and communicating efficiently in order to make possible the integration of individual knowledge to support shared knowledge and a common understanding of the design task. Analogy showed to be useful to transmit design ideas among team members (Eckert and Stacey, 2000). Relating ideas to analogical sources was seen as a powerful and effective way of 
communication. Moreover, analogy was found to be commonly used by design teams to support a wide number of design activities such as problem identification, explanations, solution generation, and knowledge communication (Ball and Christensen, 2009; Christensen and Schunn, 2007). Research on analogical thinking is extensive throughout the cognitive science literature. However, not many studies were carried out to investigate the use of this strategy in design teamwork. Therefore, in this paper we explore how the use of verbal and visual analogy contributes to design problem solving by design teams.

A major concern architectural design education is how to support the acquisition of knowledge and development of expertise in design problem solving, mainly in the conceptual phase of the design process. In a typical design studio session, students interact with their teachers, who review their projects as they convey their own design experiences and knowledge. However, major problems of this teaching model are over-reliance of students on teachers' opinions (Kurt, 2009), the imposition of a hidden agenda, and the transmission of preconceptions about how a design problem should be solved (Ward, 1990). Thus, in this study we explore how analogy can be used individually and in team as an alternative approach for the improvement of design education.

\section{Empirical Research}

\section{Research Goals}

The first goal of this study was to explore the use and aid provided by analogies in design problem solving with regard to task-related aspects, such as definition of design problems, generation of ideas, analysis of idea solutions, clarifications and evaluations of solutions. In particular, it investigated the aid provided by analogies in the design task through two different representation modes: visual and verbal. The second goal explored what were the main design activities when using analogies in two different situations: working individually, and as a team. The final goal was to analyze the contribution of analogies to the creativity of the final design outcomes produced by the team, including aspects such as: originality, aesthetic value, functionality, elaboration, overall value, and overall creativity of the design outcomes.

\section{Participants and Set Up of the Study}

Two teams each constituted by three $\mathrm{PhD}$ students belonging to the Faculty of Architecture, Department of Urbanism at TU Delft participated in the study.

Two different conditions were given, in which participants were requested to solve design problems while they were supported by either visual analogies or verbal/text analogies. These were enacted as follows: 
Visual Analogies

In this condition, students first worked independently, and thereafter they continued their design activity working together as a team. They were given a task sheet containing general instructions, a design problem, a map and photographs of the site. In addition, they were provided with a set of four images related to different themes such as art, nature, etc. and were told that this graphic material may be considered as potential analogs for their designs. Students were supplied with a set of A3 numbered sheets of paper, and were requested to produce as many sketches as possible representing their conceptual design solutions.

\section{Verbal Analogies}

In this condition, students also worked first individually and then as part of the team. They were provided with the map and photographs of the site, the same design problem, and the instructions and requirements were formulated similar to the previous condition. However, the stimuli provided consisted in a set of four written pages, each of them related to same themes provided in the visual condition.

\section{Problem}

The team was asked to design a square in order to revitalize an awkward area. To this aim, students were requested to propose design ideas about different activities and spaces that could contribute to make the plaza a more enjoyable place. Problem requirements included the design of small human scale spaces and different levels of privacy, as well as a clear circulation layout connecting these spaces. The plaza was located at the (formal) and least used main entrance (out of 6 entrances) of the Faculty of Architecture at TU Delft.

\section{Procedure}

In both conditions students start the task working individually, and afterwards they worked as a team. The four sessions (two sessions per condition) lasted around 2hs. Each condition lasted about one hour, divided into 20-25 minutes for each of the individual and team activities, and another 10 minutes for completing questionnaires related to the design activity at the end of each session (See Table 1). In the team sessions there were 7-10 minutes to make a final solution to the design problem, including a sketch of the design, labels of main parts, and a short description of how the solution works. Students were told to think aloud as the team sessions were video-taped. The experimenter responded to eventual questions but did not intervene during the session, except to remind the participants to verbalize their thoughts. 
Table 1. Experimental Conditions

\begin{tabular}{|c|c|c|c|c|c|c|c|}
\hline \multicolumn{3}{|c|}{ Visual analogy } & \multicolumn{3}{c|}{ Verbal analogy } \\
\hline \multicolumn{2}{|c|}{ Part I } & \multicolumn{2}{c|}{ Part II } & \multicolumn{2}{c|}{ Part I } & \multicolumn{2}{c|}{ Part II } \\
\hline $\begin{array}{c}\text { Single } \\
\text { session }\end{array}$ & $\begin{array}{c}\text { Self- } \\
\text { report }\end{array}$ & $\begin{array}{c}\text { Team } \\
\text { session }\end{array}$ & $\begin{array}{c}\text { Self- } \\
\text { report }\end{array}$ & $\begin{array}{c}\text { Single } \\
\text { session }\end{array}$ & $\begin{array}{c}\text { Self- } \\
\text { report }\end{array}$ & $\begin{array}{c}\text { Team } \\
\text { session }\end{array}$ & $\begin{array}{c}\text { Self- } \\
\text { report }\end{array}$ \\
\hline
\end{tabular}

\section{Results}

This exploratory study involves two teams of three students each, and therefore there is no intention to generalize results. Nevertheless, in order to find out the general impact of the main outcomes, a comparison between and within the different conditions will be carried out, and quantitative results will be presented through mean scores.

Aid Provided by the Use of Analogy in Design Problem Solving

Figure 2 shows the mean scores obtained by the aid provided by analogy, in the different design activities, when working individually and as a team, independently if students were requested to use visual or verbal analogy. It was observed that the help provided by the use of analogy in the individual sessions was highest in idea generation and problem definition, whereas in the team sessions were highest in idea generation and evaluations. The help offered by analogy in each session type showed that whereas more idea generation, problem definitions, and solution analyses were produced individually, more evaluations and clarifications were carried out when working in a team.

Figure 2. Mean Scores for Design Activities Characteristic in Individual and Team Sessions when Using Analogy

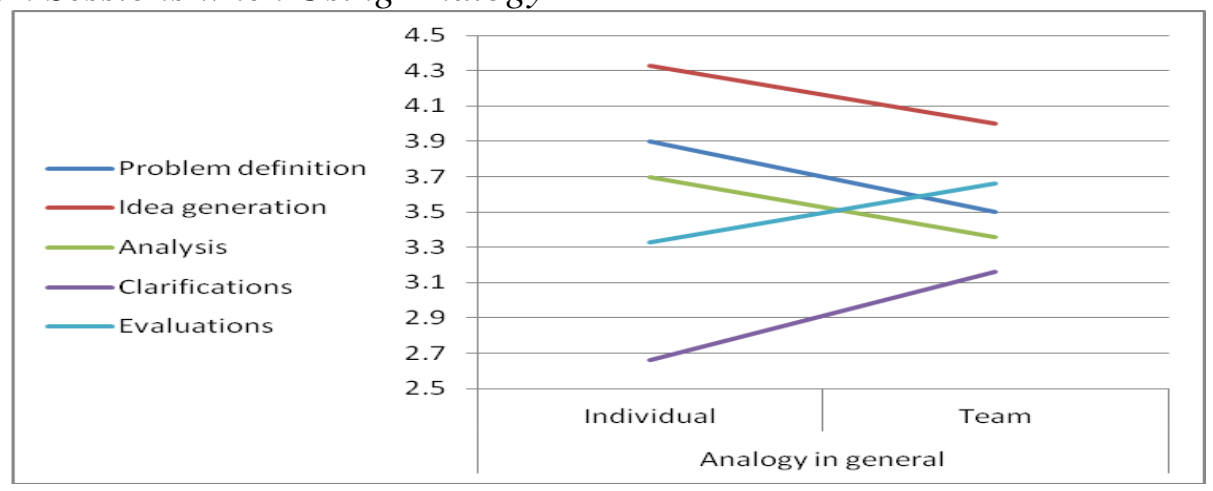

Results in both the individual and team sessions confirmed previous findings about the important role of analogy as an efficient cognitive mechanism for the generation of a large number of ideas, mainly in the conceptual phases of the design process (Casakin, 2004). When working individually, the use of analogy also showed to assist in the definition of problems, an aid that is critical in ill-defined problem solving. Considering that designers started the task working alone, when they joined the team they used 
analogy not only to continue generating design ideas, but also to raise design aspects that remained unclear (Ball and Christensen, 2009), and finally to evaluate the feasibility of solution ideas before producing a final solution.

Figure 3 shows the mean scores obtained by the aid provided by visual analogy and verbal analogy in the different design activities, irrespectively if designers were working individually or as a team. It was observed that the help provided by the use of visual analogy was highest in idea generation and problem definition, whereas the aid by verbal analogy was highest in idea generation and analysis of solutions. A comparative analysis between the two tools showed that while visual analogy was highest in problem definition, clarifications of ideas, and solution evaluations, verbal analogy were highest in idea generation. No differences were observed for analysis of solutions.

Findings showed that disregarding the representation mode used, the first and foremost role of analogy in design problem solving is concerned with idea generation. This is in line with previous research showing the powerful effect of analogies as sources of inspiration, mainly in the conceptual phases of the design process (Casakin, 2012; Goncalves et al., 2014). That verbal analogy was highest in idea generation can be explained by the fact that by being more abstract, it can endow the designer with high freedom to transcend familiar knowledge, and thus be less constrained to produce a higher number of idea solutions. On the other hand, problem definition, clarifications, and solution evaluations are all design actions that can be supported by concrete examples, such as those offered by visual analogies. Establishing mappings between the visual sources and the design problem can be helpful to frame and explain the design situation, as well as to assess the design outcome in terms of the analogical source.

Figure 3. Mean Scores for the Aid Provided by Visual and Verbal Analogy in the Design Sessions

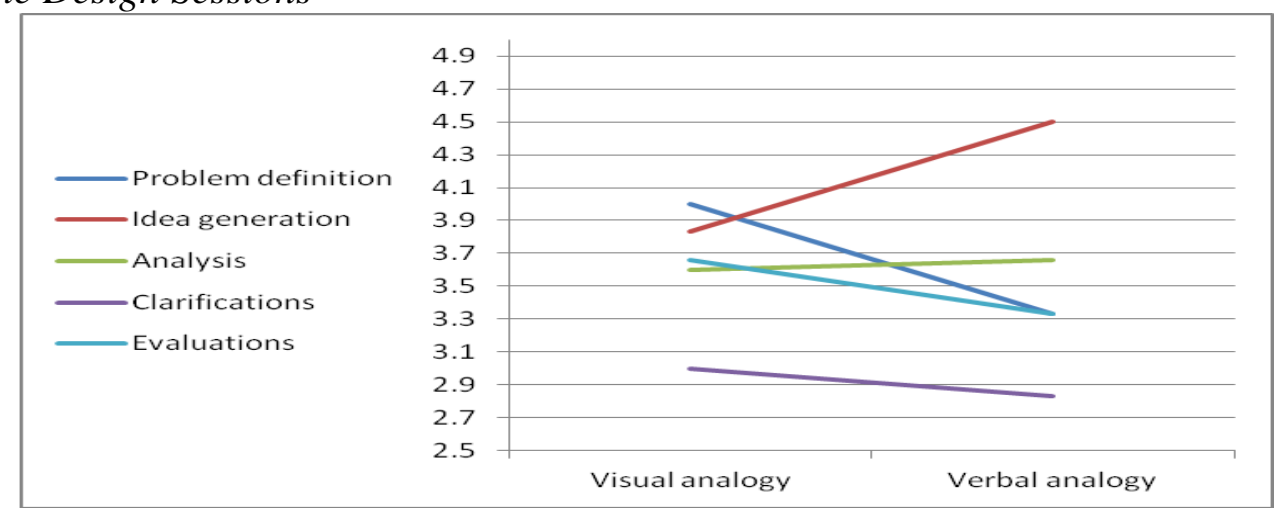

Aid Provided by Visual and Verbal Analogies in Individual and Team Sessions

Figure 4 depicts the mean scores obtained by the groups provided with visual analogies and verbal analogies in the different design activities, when working individually and as a team.

Observing the visual analogy condition, it was found that the help provided by the use of visual analogies to the designers working individually 
was highest in problem definition and idea generation, medium in analysis and evaluations, and lowest in clarifications. On the other hand, the assistance provided by this tool for the team was highest in problem definition, medium in idea generation, analysis and evaluations, and lowest in clarifications. When comparing the help provided by the use of visual analogies in the individual and team sessions, it was found that more ideas were produced individually, and more clarifications were made when working in team. The help for the other design activities did not vary.

In the verbal condition, it was observed that the aid provided by the analogies to the designers working individually was highest in idea generation, medium in solution analysis and problem definition, and lowest in evaluations and clarifications. In the team session, verbal analogies were highest in idea generation, medium in analysis and evaluations, and lowest in problem definition and clarifications. While comparing the aid provided by the use of verbal analogies in the individual and team sessions, it was observed that whereas more ideas, analysis and problem definitions were generated when working individually, more clarifications and evaluations were generated by the team.

Comparing the processes of each group related to the two instructions of using visual or verbal analogies, it was found that in the individual sessions the assistance of visual analogy scored higher in problem definition and solution evaluations, whereas the help of verbal analogy was higher in the generation of ideas and frequency of analysis. No differences were found between the experimental conditions for the amount of clarifications. Moreover, in the team sessions, designers using visual analogy scored higher in problem definition and amount of clarifications, whereas the group using verbal analogy scored higher in idea generation. No significant differences were found between both experimental conditions for clarifications and analysis of solutions.

Remarkable differences were found between the two experimental conditions, in which designers were instructed to use either visual or verbal analogy. Findings from the individual session of the visual analogy condition confirms once again previous findings about the important role of analogy as an efficient cognitive mechanism for the generation of a large number of ideas, mainly in the conceptual phases of the design process (Christensen and Schunn, 2007). When working individually, the use of analogy also showed to assist in the definition of problems, which is critical in ill-defined design problem solving characterized by no clear goals and fuzzy initial conditions (Simon, 1984). Analysis, clarifications, and evaluations of design solutions were probably unnecessary at this early stage of the design process, and therefore the contribution of analogy to these activities was lower.

When design students worked in team, they used analogies to continue structuring the design problem. Probably the interactions maintained with other team members were useful to restructure the ill-defined problem anew. It is interesting that idea generation was higher when working alone. Possibly, designers are more fluent in ideation when they do not have to explain or negotiate their ideas with others, or when their ideas are not judged by other 
team members. On the other hand, working in team enabled to use visual analogies to expose different views, and by so doing clarify problematic issues of the design.

While comparing the aid provided by visual and verbal analogies, it was observed that in the individual sessions visual analogy scored higher in problem definition and solution evaluations, whereas verbal analogy scored higher in the generation of ideas and frequency of analysis. It seems reasonable to suggest that visual analogies, which are represented through concrete examples, enable to establish clear correspondences with the design problem (Casakin, 2010; Casakin and Goldschmidt, 2000). For this reason they played an important role in the framing and structuring of the design problems, as well as in the evaluation of the idea solutions. Verbal analogies, on the other hand, are more abstract and they guided the idea generation process without constraining it as much as the concrete examples retrieved from the visual analogies did.

Almost similar results were found in the team sessions, where the group employing visual analogy scored higher in problem definition and amount of clarifications; likewise the group using verbal analogy scored higher in idea generation. These findings reinforce the significant input that visual analogies have in understanding, framing, and constraining the design problem on one hand, and the critical role of verbal analogies in helping students to 'think-outof-the box', and enlarge the production of a large number of design ideas to a farther limit.

Figure 4. Mean Scores for the Aid Provided by Visual Analogy and Verbal Analogy in Individual and Team Sessions

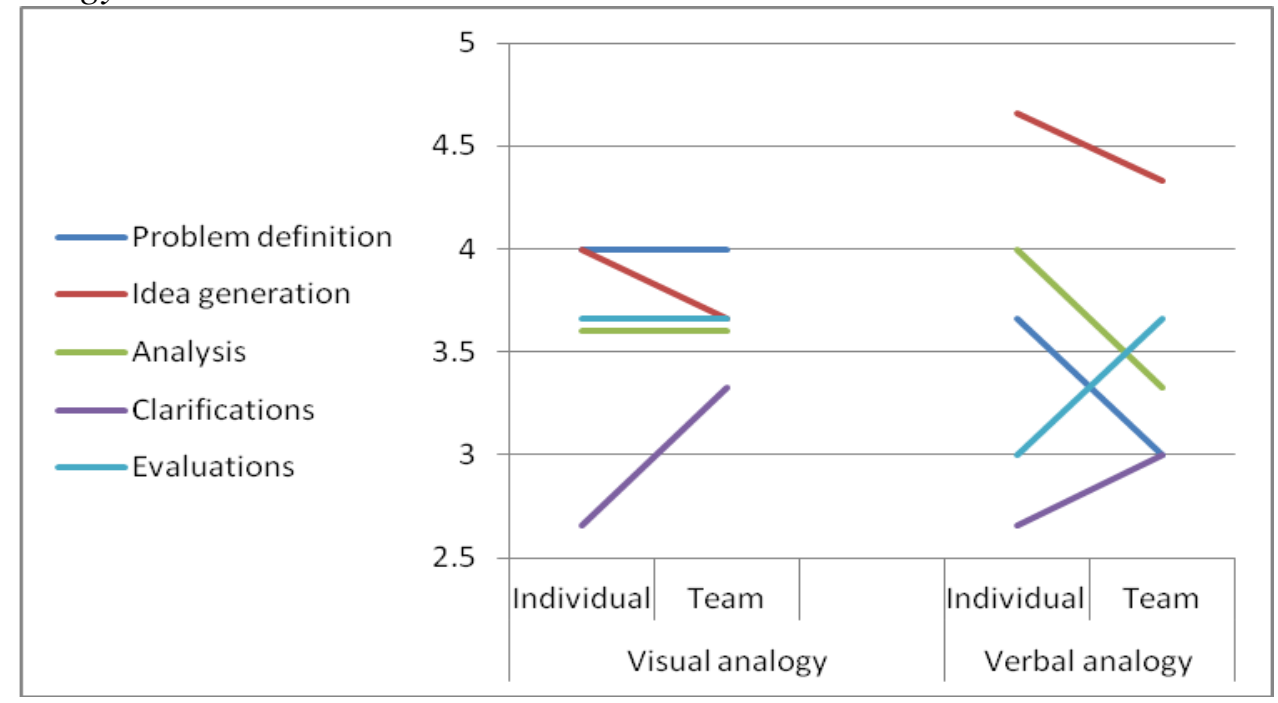

Visual and Verbal Analogies, and Creativity

Results showed that whereas the use of visual analogy scored higher for functionality of the design (3.66 over 3), small or no differences where found for originality, aesthetic value, elaboration, overall value, and overall creativity. The major contribution of both tools was in the aesthetic value (4.33 
for visual analogy and 4 for verbal analogy), and originality of the final outcome (4 for visual analogy and 3.66 for verbal analogy).

This last finding supports previous studies indicating that above anything else, analogizing is a cognitive mechanism with a strong input in originality, considered as the most representative aspect of creativity.

\section{Conclusions}

This study dealt with the role played by analogy in design problem solving, specifically in the process and outcomes produced in two design sessions. We analyzed the differences in terms of design activities, when students work individually and in team. We also examined the most frequent design activities developed along the process when using visual and verbal analogies. Finally, we investigated the contribution of both tools on the creativity of the final design outcome.

As alternative to traditional design approaches, the use of visual and verbal analogies were found to be useful tools that aided students in different ways to develop and extend their knowledge structures in the architectural design domain (Casakin, 2010; 2012). Findings of this investigation have important implications for design education. Preliminary findings showed that the use of visual analogy seems to support a more detailed definition of the problem, more clarifications of ideas, and frequent evaluations of solutions. On the other hand, verbal analogy tends to support the generation of a large number of innovative ideas, and by this to enhance design creativity. Design studio teachers interested in implementing intervention programs aimed at supporting students along the design process should consider the present findings. Since the way that external analogical sources are represented showed to have a different effect on the various activities of the design process, as well as on the modality of work, a major challenge will be to know how and when to integrate the use of abstract and concrete displays in an optimal way for supporting individual and team activity during the different phases of the design process.

\section{References}

Ball, L. J., and Christensen, B. T. 2009. Analogical reasoning and mental simulation in design: two strategies linked to uncertainty resolution, Design Studies, 30, 169186.

Ball, L. J., Ormerod, T. C., and Morley, N. J. 2004. Spontaneous analogising in engineering design: a comparative analysis of experts and novices. Design Studies, 25, 495-508.

Bearman, C. R., Ball, L. J., and Ormerod, T. C. 2007. The structure and function of spontaneous analogising in domain-based problem solving. Thinking \& Reasoning, 13, 273-294. 
Brown, A. 1989. Analogical learning and transfer: What develops?, In Similarity and Analogical Reasoning, S. Vosniadou, and A. Ortony, Eds. Cambridge University Press, Cambridge, 369-411.

Cai, H., Yi-Luen Do, E., and Zimring, C. M. 2010. Extended linkography and distance graph in design evaluation: an empirical study of the dual effects of inspiration sources in creative design. Design Studies, 31, 146-168.

Cardoso, C., and Badke-Schaub, P. 2011. The influence of different pictorial representations during idea generation. The Journal of Creative Behavior, 45, 130-146.

Casakin, H. 2004. Visual analogy as a cognitive strategy in the design process: Expert versus novice performance. Journal of Design Research on Design Expertise, 4 (2).

Casakin, H. 2010. Visual analogy, visual displays, and the nature of design problems: the effect of expertise. Environmental Planning and Design: Design B., 37, 170188.

Casakin, H. 2012.Visual analogy as a cognitive stimulator for idea generation in design problem solving. In The Psychology of Problem Solving: An Interdisciplinary Approach. S. Helie, Ed. Nova Science Publishers: New York.

Casakin, H., and Goldschmidt, G. 2000. Reasoning by visual analogy in design problem-solving: the role of guidance. Environment and Planning B: Planning and Design, 27, 105-119.

Christensen, B. T., and Schunn, C. D. 2007. The relationship of analogical distance to analogical function and pre-inventive structure: The case of engineering design, Memory and Cognition, 35, 29-38.

Dunbar, K., and Blanchette, I. 2001. The in vivo/ in vitro approach to cognition: the case of analogy, Trends in Cognitive Sciences, 5, 334-339.

Eckert, C. M., and Stacey, M. K. 2000. Sources of inspiration: A language of design. Design Studies, 21, 523-538.

Gentner, D., and Markman, A. B. 1997. Structure mapping in analogy and similarity. American Psychologist, 52, 45-46.

Goel, V., and Pirolli, P. 1992. The structure of design problem spaces. Cognitive Science, 16, 395-429.

Goncalves, M.; Cardoso, C.; Badke-Schaub, P. 2014, What inspires designers? Preferences on inspirational approaches during idea generation. Design Studies, $35,1,29-53$.

Goldschmidt, G. 1995. Visual displays for design: Imagery, analogy and databases of visual images. In Visual Databases in Architecture. A. Koutamanis, H. Timmermans, and I. Vermeulen, Eds. Aldershot, Avebury, 53-74.

Goldschmidt, G., and Smolkov, M. 2006. Variances in the impact of visual stimuli on design problem solving performance. Design Studies, 27, 549-569.

Goldschmidt, G., and Litan Sever, A. 2011. Inspiring design ideas with texts. Design Studies, 32, 139-155.

Hannington, B. 2003. Methods in the making: a perspective on the state of human research in design. Design Issues, 19, 9-18.

Holyoak, K. J., and Thagard, P. 1996. Mental leaps: analogy in creative thought. Cambridge, MA: MIT Press.

Holyoak, K. J., and Thagard, P. 1997. The analogical mind. American Psychologist, $52,35-44$.

Hofstadter, D. R. 2001. Epilogue: Analogy as the core of cognition. In The Analogical Mind : Perspectives from Cognitive Science, D. Gentner, K. L. Holyoak, and B. N. Kokinov, Eds. MIT Press, Cambridge, MA, 499-538. 
Koestler, A. 1964. The sleepwalkers: A history of man's changing vision of the world. Penguin Books, Hardmondsworth.

Kuhn, T.S. 1970. The structure of scientific revolutions, University of Chicago Press, Chicago.

Kurt, S. 2009. An analytic study on the traditional studio environments and the use of the constructivist studio in the architectural design education. Procedia Social and Behavioral Sciences, 1, 401-408.

Malaga, R. A. 2000. The effect of stimulus modes and associative distance in individual creativity support systems. Decision Support Systems, 29, 125-141.

Perttula, M., and Sipila, P. 2007. The idea exposure paradigm in design idea generation. Journal of Engineering Design, 18, 93-102.

Schon, D. 1966. Displacement of Concepts. Humanities Press, New York.

Simon, H. 1984. The structure of ill-structured problems. In: Developments in Design Methodology, N. Cross, Ed.. John Wiley and Sons, New York, 145-165.

Visser, W. 1996. Use of episodic knowledge and information in design problem solving. In Analysing Design Activity, N. Cross, H. Christiaans, and K. Dorst, Eds. Wiley, Chichester, 271-289.

Ward, A. 1990. Ideology, culture and the design studio. Design Studies, 11, 10-16. 
